\title{
A formalization of one of the main claims of "Cortex reorganization of Xenopus laevis eggs in strong static magnetic fields" by Mietchen et al. $2005^{1}$
}

\author{
Daniel Mietchen
}

Fraunhofer Institute for Biomedical Engineering (IBMT), Sulzbach, Germany

E-mail: daniel.mietchen@ibmt.fraunhofer.de; ORCID: https://orcid.org/0000-0001-9488-1870

Editor: Cristina-Iulia Bucur (https://orcid.org/0000-0002-7114-6459)

Review comments from: Michel Dumontier (https://orcid.org/0000-0003-4727-9435); Tobias Kuhn

(https://orcid.org/0000-0002-1267-0234); Cristina-Iulia Bucur (https://orcid.org/0000-0002-7114-6459)

Received 25 July 2021

Accepted 17 November 2021

Abstract. Mietchen et al. claimed in previous work that strong static magnetic fields change the cell cortex in dejellied fertilizable stage VI Xenopus lavis oocytes. We present here a formalization of that claim, stating that all things of class "strong static magnetic field" that are in the context of a thing of class "dejellied fertilizable stage VI Xenopus laevis oocyte" generally have a relation of type "affects" to a thing of class "cell cortex" in the same context.

Keywords: Dejellied fertilizable stage VI Xenopus laevis oocyte, strong static magnetic field, cell cortex

\section{Introduction}

Mietchen et al. [2] state that "A complex reorganization of cortical pigmentation was found in dejellied eggs as a function of the magnetic field and the field exposure time". We present here a formalization of the main scientific claim from this quote by using a semantic template called the super-pattern [1].

\section{Formalization}

Our formalization looks as follows:

\footnotetext{
${ }^{1}$ As RDF/nanopublication: http://purl.org/np/RAXVRaFjWDIX5cZcVRXETaEIAx6QAyLK5JCrzDP-yDp9U 
CONTEXT-CLASS ("in the context of all ..."): SUBJECT-CLASS ("things of type ..."):

QUALIFIER:

RELATION-TYPE ("have a relation of type..."):

OBJECT-CLASS (“to things of type...”): dejellied fertilizable stage VI Xenopus laevis oocyte strong static magnetic field generally

affects

cell cortex

In the context class, we use the class "dejellied fertilizable stage VI Xenopus laevis oocyte" (Q107644116) from Wikidata. In the subject class, we use the class "strong static magnetic field" (Q107644241) from Wikidata. In the object class, we use the class "cell cortex" (Q5058180) from Wikidata.

\section{RDF code}

This is our formalization as a nanopublication in TriG format:

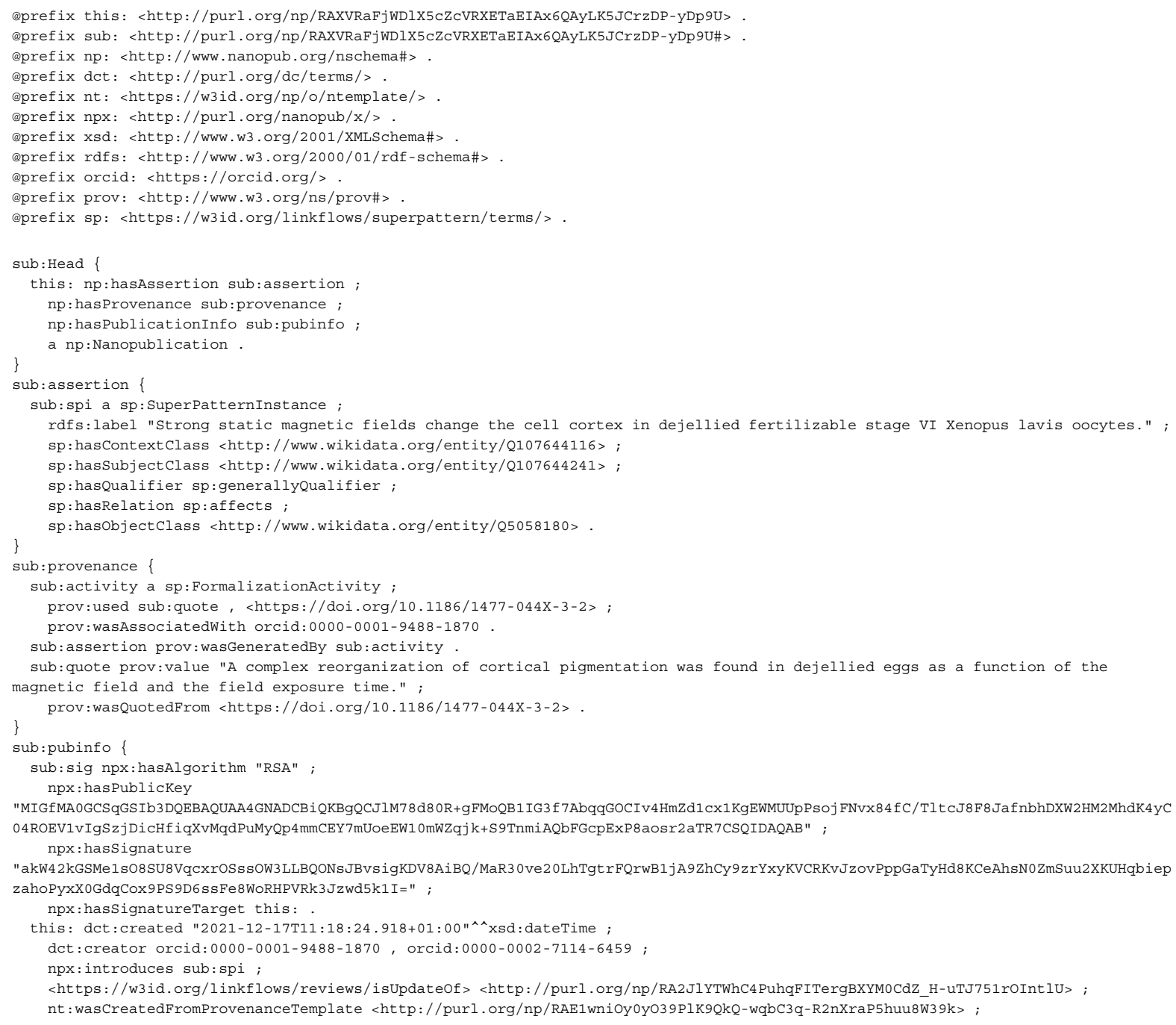


nt: wasCreatedFromPubinfoTemplate <http://purl org/np/RA2vCBXZf-icEcVRGhulJXugTGxpsV5yVr9yqCI1boh4A> , <http://purl.org/np/RAA2MfqdBCzmz9yVWj KLXNbyfBNCwSMmOqCNUxkk1maIM>

<http://purl.org/np/RAOGu9Lh0BD4tbIRB9RG6RGRA_ObDh75NTbIqaWgxxs8M>， <http://purl.org/np/RAWv_eqe4tghgOOg6NqRQODj C865Q0ZWkXTXqjSe59Y4> ;

nt: wasCreatedFromTemplate <http://purl.org/np/RAv68imZrEjfcp2rnEg1hzoBqEVc0cQMtp9 1Za0BxNM4 > .

\section{References}

[1] C.I. Bucur, T. Kuhn, D. Ceolin and J. van Ossenbruggen, Expressing high-level scientific claims with formal semantics, in: Proceedings of the 11th Knowledge Capture Conference, 2021. doi:10.1145/3460210.3493561.

[2] D. Mietchen, J.W. Jakobi and H.P. Richter, Cortex reorganization of Xenopus laevis eggs in strong static magnetic fields, BioMag Res Tech 3 (2005), 2. doi:10.1186/1477-044X-3-2. 